\title{
Validating the use of short interfering RNA as a novel technique for cell-specific target gene knockdown in lung ischemia-reperfusion injury
}

\author{
Heather E. Merry, MD, ${ }^{a}$ Patrick Phelan, MD, ${ }^{a}$ Billanna Hwang, MPH, ${ }^{a, b}$ and Michael S. Mulligan, MD ${ }^{\mathrm{a}, \mathrm{b}}$
}

\begin{abstract}
Objective: Short interfering RNA is an effective method for target gene knockdown. However, concerns surround the design, administration, efficacy, specificity, and immunostimulatory potential. Although uptake by alveolar macrophages has been demonstrated, studies have not examined its use in lung ischemia-reperfusion injury. We describe the validation of short interference RNA as a novel technique for cell-specific target gene knockdown in our model of lung ischemia-reperfusion injury.
\end{abstract}

Methods: Dose-response experiments were performed, and 3 distinct sequences of toll-like receptor-4, toll-like receptor-2, and myeloid differentiation factor-88 short interference RNA were tested for efficacy of knockdown. Saline, lipid vector, and noncoding short interference RNA controls were used. Similar experiments were performed in primary cultures of resident pulmonary cells. Target protein knockdown was assessed by Western blot. Rat serum and cell culture media were assessed for interferon and cytokine production. Biotin labeling was used to assess short interference RNA uptake.

Results: Target protein expression was significantly reduced using short interference RNA. However, toll-like receptor-4 knockdown was isolated to alveolar macrophages, and biotin labeling confirmed toll-like receptor-4 short interference RNA localization to alveolar macrophages. There was significant knockdown of toll-like receptor-4 expression in cultured cells treated with toll-like receptor-4 short interference RNA. There was no significant change in interferon production after short interference RNA treatment. There was effective target protein knockdown with each sequence used.

Conclusions: Short interference RNA is a valid method for achieving target protein knockdown in alveolar macrophages and is an important tool in the evaluation of its role in the development of lung ischemia-reperfusion injury. ( $\mathrm{J}$ Thorac Cardiovasc Surg 2016;151:499-506)

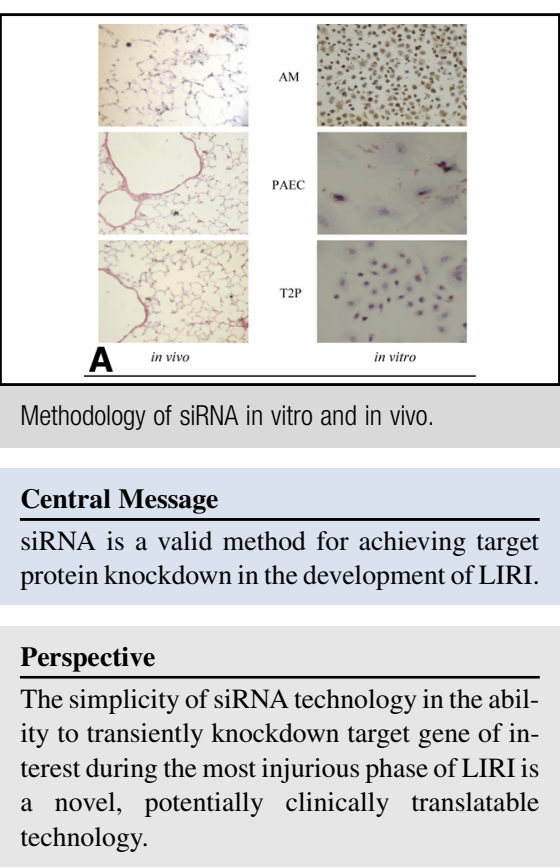

See Editorial Commentary page 507.

\footnotetext{
From the aivision of Thoracic Surgery, University of Washington, Seattle, Wash; and ${ }^{\mathrm{b}}$ University of Washington, Center for Lung Biology, Seattle, Wash.

Funded by the National Institutes of Health (Bethesda, Md) Grant R01HL093097 and RO1-Role of TLR-4 in Lung Reperfusion Injury. The content is solely the responsibility of the authors and does not necessarily represent the official views of the National Institutes of Health or its subsidiary institutes and centers.

Received for publication Feb 18, 2015; revisions received Sept 4, 2015; accepted for publication Sept 5, 2015; available ahead of print Dec 11, 2015.

Address for reprints: Billanna Hwang, MPH, Division of Thoracic Surgery, University of Washington, Center for Lung Biology 850 Republican St, Box 358052, Seattle, WA 98109 (E-mail: bhwang@uw.edu).

$0022-5223 / \$ 36.00$

Copyright (c) 2016 by The American Association for Thoracic Surgery

http://dx.doi.org/10.1016/j.jtcvs.2015.09.125
}

RNA interference with short interfering RNA (siRNA) is a powerful research tool for the selective, transient posttranscriptional knockdown of gene expression both in vivo and in vitro. ${ }^{1-3}$ siRNA consists of short, doubled-stranded RNA duplexes, 21 to 25 nucleotide base pairs in length, designed to specifically target and cleave the mRNA of the gene of interest, thereby transiently preventing protein expression of the target gene. ${ }^{4}$ Several distinct advantages with siRNA use include its efficacy and specificity, versatile array of prospective targets, and broad therapeutic potential. ${ }^{2,4,5}$ Although 


$$
\begin{aligned}
& \text { Abbreviations and Acronyms } \\
& \begin{aligned}
\text { AM } & =\text { alveolar macrophage } \\
\text { CINC } & =\text { cytokine-induced neutrophil } \\
& \text { chemoattractant } \\
\text { IFN } & \text { interferon } \\
\text { LIRI } & =\text { lung ischemia-reperfusion injury } \\
\text { MyD88 } & \text { myeloid differentiation factor- } 88 \\
\text { OTE } & =\text { off-target effect } \\
\text { PAEC } & =\text { pulmonary artery endothelial cell } \\
\text { siRNA } & =\text { short interfering RNA } \\
\text { TLR } & =\text { toll-like receptor } \\
\text { T2P } & =\text { type } 2 \text { pneumocyte }
\end{aligned}
\end{aligned}
$$

in vivo siRNA use has been studied in models targeting multiple organ systems, including the lung, it has yet to be comprehensively validated in a model of lung ischemia-reperfusion injury (LIRI). Many in vitro studies have shown the diverse applicability and success of siRNA in multiple cell lines, whereas in vivo translation has been impaired in part by the lack of cell-specific targeting, particularly endothelial and epithelial siRNA transfection. ${ }^{6-8}$ Having previously identified the central importance of the alveolar macrophage (AM) in LIRI, ${ }^{9}$ it would be highly desirable if we were effective in selectively transfecting the AM in vivo. Intravenously administered siRNA has been shown to localize to resident tissue inflammatory cells, including Kupffer cells in the liver, peritoneal macrophages, and AM in the lung. ${ }^{7,10-12}$ Therefore, we were optimistic that we would be successful in transfecting the AM in the lung in our model of LIRI.

LIRI remains a significant clinical problem after lung transplantation in up to $25 \%$ of recipients and increases the risks of acute and chronic rejection and bronchiolitis obliterans, and has been found to adversely affect early post-transplant mortality. ${ }^{13-16}$ Understanding the cell-specific inflammatory signaling mechanisms relevant to LIRI may lead to the identification of strategies aimed at alleviating this form of lung injury. Our warm ischemia model of LIRI in the rat is not only relevant but also provides for the evaluation of LIRI outcomes with a predictable time of onset that may allow for practical pretreatment. Our laboratory and others have identified several site-specific mediators and characterized the central importance of early activation of the AM in the development of LIRI. ${ }^{9,17,18}$ In addition, toll-like receptor (TLR) 4, TLR2, and myeloid differentiation factor-88 (MyD88) have emerged as potential upstream regulators of the inflammatory response in LIRI. ${ }^{19,20}$ The predictable time of onset and transient nature of siRNA administration make it an attractive tool for in vivo examination of the mechanistic role these upstream regulators have in the development of LIRI.

Despite widespread adoption of RNA interference, there are several concerns related to its use. Some authors urge caution when interpreting results from experiments that have not addressed what is broadly referred to as off-target effects (OTEs). ${ }^{21,22}$ Specifically, OTEs include silencing of nontarget genes, activation of nonspecific immune responses, notably the interferon (IFN) response, toxicity, and saturation of a cell's native mRNA processing, thereby affecting its ability to function normally. ${ }^{21,22}$ siRNA manufacturers have developed strategies to deal with some aspects of OTEs, such as siRNA design and control siRNA, but they are independent of individual experimental conditions and model systems.

The purpose of this study was to develop a comprehensive approach to validate the novel use of siRNA in vivo in our well-developed warm ischemia model of LIRI in the rat and in vitro in our cell culture-based model of hypoxia and reoxygenation. Our strategy includes consideration and evaluation of siRNA design, dose response, uptake or localization, and efficacy and specificity, particularly focusing on minimizing OTEs, such as the IFN response.

\section{MATERIALS AND METHODS \\ Reagents}

All reagents were purchased from Sigma Chemical Company (St Louis, Mo) unless otherwise specified.

\section{Short Interfering RNA Design and Transfection}

The siRNAs used in this study were obtained from Invitrogen (Carlsbad, Calif). Three unique siRNA duplexes were designed to target TLR2, TLR4, and MyD88 mRNA. Sequences containing $5^{\prime}$-UGUGU-3' or $5^{\prime}$ GUCCUUCAA- $3^{\prime}$, which are known to activate the innate immune response (IFN production), were not incorporated. ${ }^{23}$ In addition, scrambled, noncoding (nonsense) siRNA that contains limited sequence homology to rat, mouse, or human genome served as control siRNA. For in vivo transfection, siRNA was administered in 2 equally divided doses 24 and 48 hours before undergoing the in situ ischemia-reperfusion protocol. An amount of $10 \mathrm{nM}$ of siRNA (based on dose-response experiments) was diluted in $250 \mu \mathrm{L}$ of sterile saline and combined with $100 \mu \mathrm{L} / \mathrm{kg}$ of lipofectamine 2000 (Invitrogen) diluted in $250 \mu \mathrm{L}$ of sterile saline. After 15 minutes of incubation, the lipid-associated siRNA was rapidly injected into the penile vein. For in vitro AM transfection, 100 pmol of siRNA was diluted in $50 \mu \mathrm{L}$ of growth media per well and incubated for 15 minutes. This was then mixed with $3 \mu \mathrm{L}$ of lipofectamine diluted in $50 \mu \mathrm{L}$ of growth media. The cell culture media was then changed and supplemented with siRNA-lipid mixture. The cells were incubated with the primer for at least 6 hours, after which the media was changed. Type 2 pneumocyte (T2P) and pulmonary artery endothelial cell (PAEC) siRNA transfection was as described earlier for AM except that $1 \mu \mathrm{L}$ of lipofectamine was used. Additional animals and cells were treated with biotinlabeled TLR4 siRNA and assessed for localization and uptake after 2 and 6 hours, respectively. 

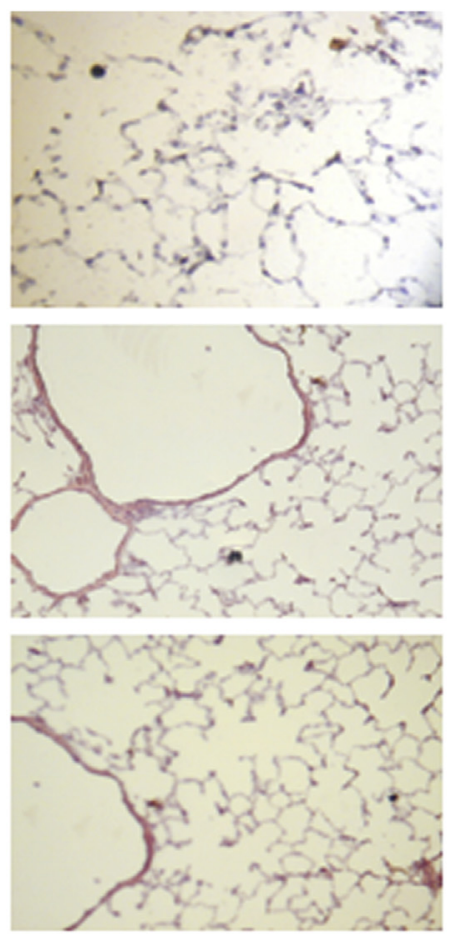

A

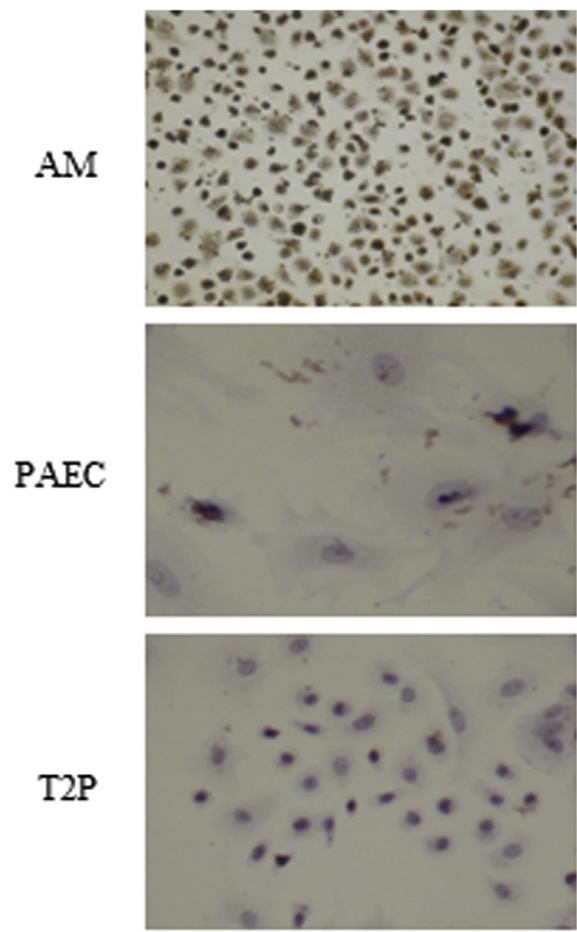

in vitro

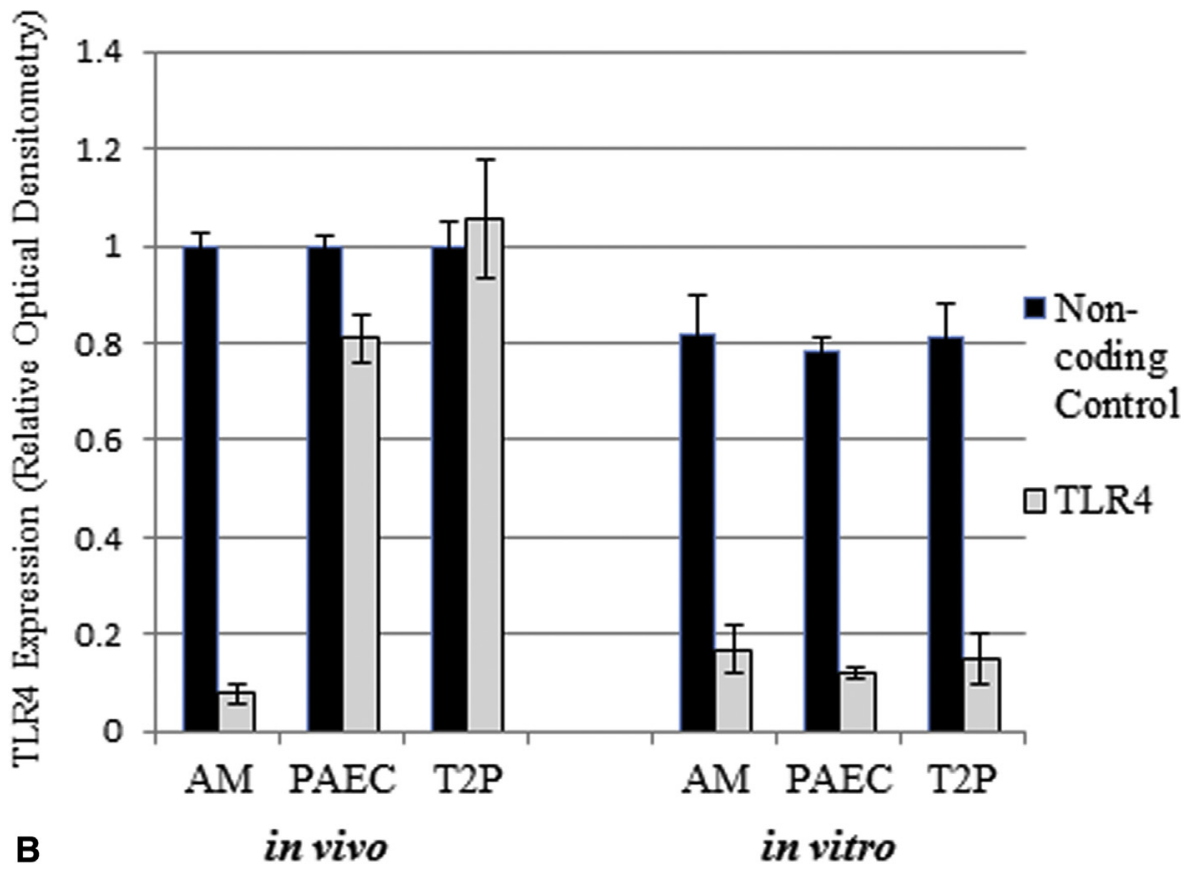

FIGURE 1. A, Localization and uptake of TLR4 siRNA. Immunohistochemistry on lung sections after in vivo biotin-labeled TLR4 siRNA administration demonstrated siRNA localization only to the AM 2 hours after treatment $(n=4)$. Immunohistochemical analysis of AMs, PAECs, and T2Ps treated with biotin-labeled TLR4 siRNA demonstrated uptake of the biotin-labeled TLR4 siRNA by all 3 cell types within 6 hours of treatment ( $\mathrm{n}=12$ wells). B, Reduction in TLR4 expression with TLR4 siRNA. TLR4 expression after TLR4 siRNA sequence- 2 treatment in vivo was reduced only in AMs (by $92 \%, P<.001$ ), not PAECs $(19 \%$ reduction, $P>.05)$ or T2P $(6 \%$ increase, $P>.05)$ compared with noncoding siRNA controls as assessed by relative optical densitometry $(\mathrm{n}=4)$. However, there was $80 \%(P<.01), 85 \%(P<.001)$, and $82 \%(P<.01)$ knockdown of TLR4 expression with TLR4 siRNA sequence-2 treatment of cultured AMs, PAECs, and T2Ps, respectively, compared with noncoding controls siRNA ( $\mathrm{n}=12$ wells per group). AM, Alveolar macrophage; PAEC, pulmonary artery endothelial cell; $T 2 P$, type 2 pneumocytes; $T L R$, toll-like receptor. 


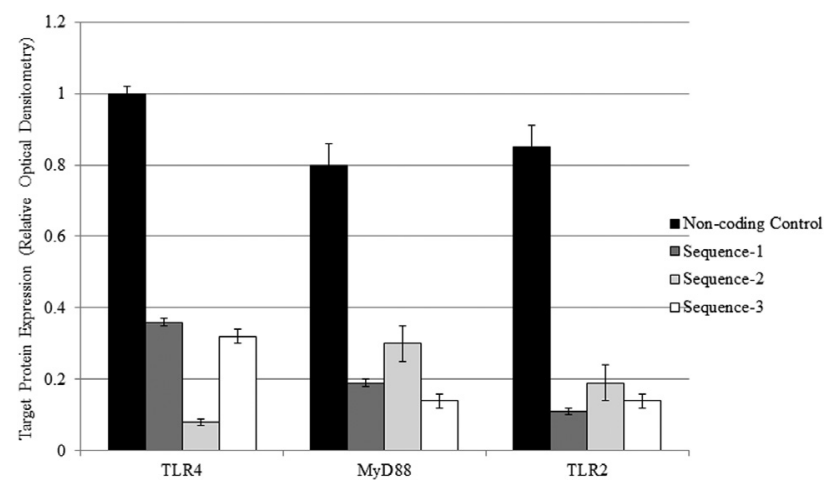

FIGURE 2. Sequence-specific knockdown efficacy. In rats pretreated with TLR4 siRNA, sequences 1,2 , and 3 , before undergoing ischemia and reperfusion, there was a $70 \%(P<.01), 92 \%(P<.001)$, and $89 \%$ $(P<.001)$ reduction, respectively, in TLR4 protein expression compared with noncoding siRNA controls as assessed by relative optical densitometry $(\mathrm{n}=3)$. Primary AM cultures treated with MyD88 siRNA 1,2 , and 3 before undergoing hypoxia and reoxygenation demonstrated $76 \%(P<.001), 63 \%(P<.01)$, and 83\% $(P<.001)$ reductions, respectively, in MyD88 expression compared with noncoding siRNA controls $(\mathrm{n}=12$ wells). Primary AM cultures treated with TLR2 siRNA sequences 1,2 , and 3 before undergoing hypoxia and reoxygenation demonstrated $87 \%(P<.001), 78 \%(P<.001)$, and $84 \%(P<.001)$ reductions, respectively, in TLR2 expression compared with noncoding siRNA controls ( $\mathrm{n}=12$ wells). TLR, Toll-like receptor; $M y D 88$, myeloid differentiation factor 88 .

\section{Ischemia-Reperfusion Protocol}

Pathogen-free adult male Long-Evans rats (Harlan Sprague Dawley, Indianapolis, Ind), weighing 275 to $300 \mathrm{~g}$, were used for all in vivo experiments. Approval for all experimental protocols was granted by the University of Washington Animal Care Committee. Animals received humane care in compliance with the "Principles of Laboratory Animal Care," established by the National Society for Medical Research, and the "Guide for the Care and Use of Laboratory Animals," developed by the Institute of Laboratory Animal Resources and published by the National Institutes of Health (NIH Publication No. 86-23, revised 1996). A well-established, in situ, warm rat ischemia-reperfusion model was used with 90 minutes of ischemia and 4 hours of reperfusion. ${ }^{9,18}$ At the end of reperfusion, a midline laparotomy and sternotomy were performed for blood sampling and excision of the heart-lung block.

\section{Cellular Harvest and Hypoxia-Reoxygenation}

AMs were isolated and cultured as we have described previously. ${ }^{24}$ Cell counts and viability were assessed by standard trypan blue exclusion methods. Cells were plated at a density of 1,000,000 cells/well and allowed to quiesce overnight. T2Ps were harvested as described previously by Dobbs. ${ }^{25}$ PAECs were isolated, cultured, and purified as described previously by our group. ${ }^{17}$ Hypoxia and reoxygenation were performed as previously described. ${ }^{24}$ In brief, once T2Ps or PAECs reached confluence or AMs have quiesced for at least 6 hours, the cells were placed in a hypoxic chamber $\left(\mathrm{FIO}_{2} 0.5 \%\right)$ for 90 minutes. The media equilibrated with the hypoxic environment in less than 5 minutes. Cells were then removed from the hypoxic chamber and placed in a normoxic $\left(\mathrm{FIO}_{2}\right.$ $21 \%$ ) incubator for up to 4 hours. Negative controls remained in the normoxic incubator for up to 6 hours.

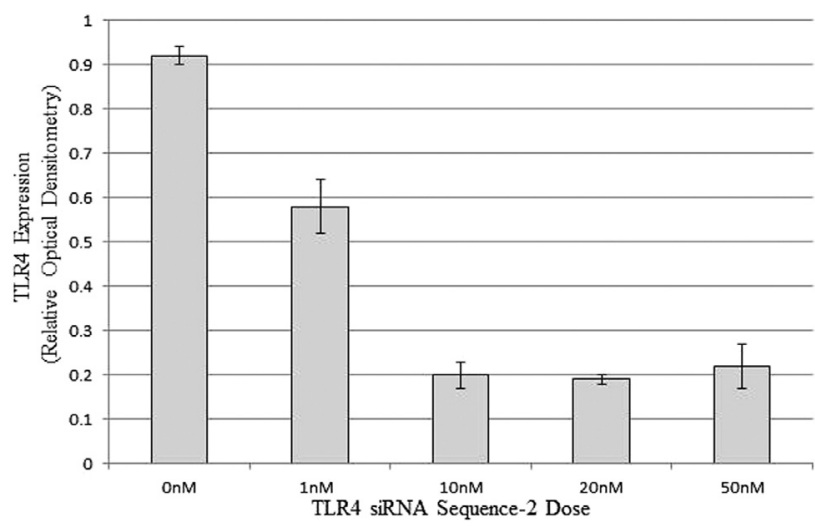

FIGURE 3. siRNA dose response. TLR 4 siRNA sequence- 2 treatment in concentrations of $0,1,10,20$, and $50 \mathrm{nM}$ reduced TLR4 expression by $8 \%$ $(P>.05), 42 \%(P>.05), 80 \%(P<.01), 81 \%(P<.001)$, and $76 \%$ $(P<.01)$, respectively, compared with noncoding siRNA controls 48 hours after the initial intravenous TLR4 siRNA administration $(\mathrm{n}=3) . T L R$, Toll-like receptor; siRNA, short interfering RNA.

\section{Experimental Groups}

Noncoding siRNA control animals and cells received intravenous noncoding scrambled siRNA before undergoing ischemia-reperfusion or hypoxia and reoxygenation, respectively. Negative control animals and cells did not receive siRNA treatment or undergo ischemia-reperfusion or hypoxia and reoxygenation. Separate animal and cell treatment groups received TLR2, TLR4, or MyD88 siRNA, lipid vector alone, or normal saline alone before undergoing ischemia-reperfusion or hypoxia and reoxygenation, respectively.

\section{Markers of Lung Injury}

Left lung permeability index was determined as described previously. ${ }^{9,18} \mathrm{I}^{125}$-radiolabeled bovine serum albumin (NEN Life Sciences, Boston, Mass) was intravenously injected 5 minutes before removal of the hilar clamp, and a scintillation counter was used to quantitate radioactivity. Permeability index was calculated as follows: permeability index $=$ left lung $(\mathrm{cpm}) / 1 \mathrm{~mL}$ blood $(\mathrm{cpm})$. Myeloperoxidase content was used to quantitate neutrophil accumulation in the lungs as described previously. ${ }^{9,18}$ Briefly, lung samples were homogenized and sonicated, the supernatants recovered, and the change in absorbance at $460 \mathrm{~nm}$ wavelength was recorded after mixing $50 \mu \mathrm{L}$ of each sample with $1.45 \mathrm{~mL}$ of assay buffer. Left lung bronchoalveolar lavage was performed after harvesting the heart lung block by placing a clamp across the right hilum and using the intratracheal angiocatheter to lavage the lung with $3 \mathrm{~mL}$ of cold sterile saline as described previously. ${ }^{9,18}$ The recovered lavage fluid was centrifuged at $1800 \mathrm{rpm}$ for 10 minutes at $4{ }^{\circ} \mathrm{C}$ to pellet the cells. The supernatant was frozen for cytokine analysis, and the pellet was resuspended in $10 \mathrm{~mL}$ of sterile phosphate-buffered saline. One milliliter of resuspended cells was stained with Gill's solution and counted using a hemacytometer (Hausser Scientific, Horsham, Pa).

\section{Protein Analysis for Cytokine Content by Enzyme- Linked Immunosorbent Assay}

Media from cell cultures and serum from rats pretreated with siRNA were collected 1, 6, and 24 hours after siRNA administration and processed as described earlier (serum) or as previously published (media) ${ }^{3}$ and then analyzed for IFN $\gamma$ and IFN $\beta$ content via sandwich enzyme-linked immunosorbent assay. ${ }^{24}$ Likewise, media from cell cultures were collected after undergoing hypoxia and reoxygenation and analyzed for cytokine-induced neutrophil chemoattractant (CINC) content via enzyme-linked 
Permeability Index

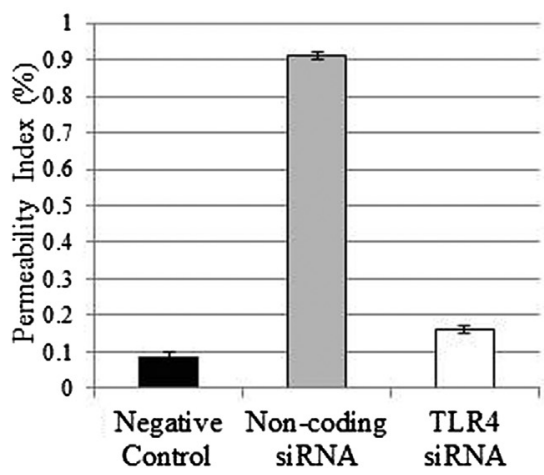

MPO Content

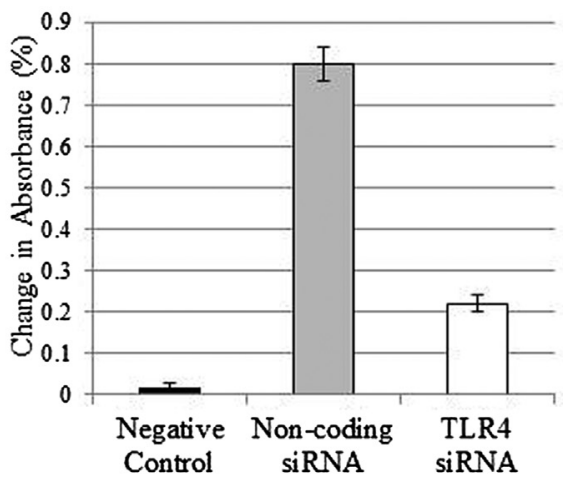

BAL Leukocytes

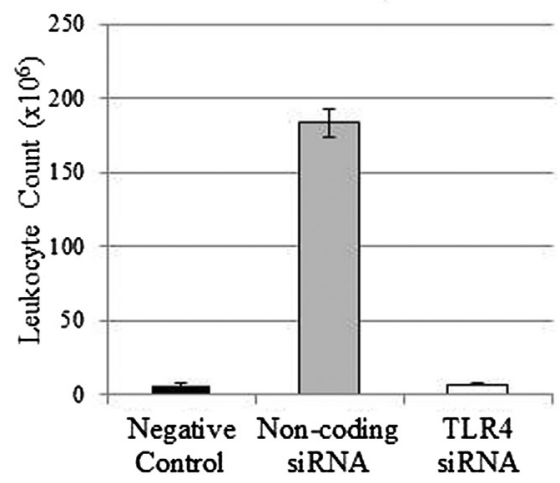

FIGURE 4. Reduced lung injury with TLR4 knockdown. Vascular permeability, myeloperoxidase content, and bronchoalveolar lavage leukocyte count were increased by 11-fold, 42-fold, and 28-fold in noncoding siRNA controls after undergoing ischemia-reperfusion compared with negative controls that did not undergo ischemia-reperfusion $(\mathrm{n}=3)$. However, with TLR4 siRNA treatment before undergoing ischemia-reperfusion, vascular permeability, myeloperoxidase content, and bronchoalveolar lavage leukocyte count were all reduced by $82 \%(P<.001), 73 \%(P<.01)$, and $96 \%(P<.001)$, respectively, compared with noncoding siRNA controls. siRNA, Short interfering RNA; TLR, toll-like receptor; $M P O$, myeloperoxidase; $B A L$, bronchoalveolar lavage.

immunosorbent assay as described. Samples and standards were run in triplicate.

\section{Western Blot Analysis}

Total protein was extracted from whole left lung homogenates and cultured cells after 15 minutes of reperfusion, and Western blot analysis was performed as previously described using $40 \mu \mathrm{g}$ of protein and TLR2, TLR4, or MyD88-specific antibody (Cell Signaling, Beverly, Mass). Densitometry was performed to assess relative differences between groups using Image J (version 1.2; Cybernetics Corp, Silver Springs, Md).

\section{Immunohistochemistry Protocol}

Whole lung tissue specimens were fixed in $4 \%$ paraformaldehyde, processed, sectioned, and stained previously described. ${ }^{12}$ Stained sections were examined using ImageJ software (Version 1.2).

\section{Statistical Analysis}

All data are presented as mean values + the standard error of the mean. Comparisons among groups were made using 1-way analysis of variance. Bonferroni's method was used to adjust for multiple comparisons. A post hoc 2-tailed Student $t$ test was performed to assess statistical differences between individual groups.

\section{RESULTS}

We developed a specific, comprehensive approach using siRNA in vivo and in vitro to characterize signaling pathways and cellular responses in LIRI. Two hours after treatment in the in vivo in situ model of lung ischemiareperfusion, an appreciable uptake of the TLR4 siRNA was present only in AMs. There was no appreciable uptake in other lung cell types, including rat pulmonary endothelial cells and T2P (Figure 1, A). However, in the primary cell culture model, uptake of the siRNA was achieved in all 3 cell lines (AM, PAEC, and T2P) within 6 hours of treatment (Figure 1, A). As expected given the differential uptake of siRNA in the in vivo model, TLR4 protein expression after TLR4 siRNA sequence-2 treatment in vivo was reduced only in AM, not PAEC or T2P. Noncoding siRNA-treated animals were used as controls, and protein expression was assessed by relative optical densitometry (Figure 1, B).

In the cultured cell lines undergoing in vitro hypoxia and reoxygenation, there was consistent $80 \%, 85 \%$, and $82 \%$ knockdown of TLR4 expression with TLR4 siRNA treatment of cultured AM, PAEC, and T2P, respectively, compared with noncoding siRNA controls (Figure 1, B). Sequence 2 of the TLR-4 siRNA was used in the experiments because it had the highest affinity and level of knockdown. Rats pretreated with different TLR4 siRNA sequences (sequences 1, 2, and 3) before undergoing ischemia-reperfusion demonstrated a $70 \%$ to $92 \%$ reduction in TLR4 expression compared with noncoding siRNA controls as assessed by relative optical densitometry (Figure 2). Primary AM cultures treated with MyD88 or TLR2 siRNA sequences 1,2 , and 3 before undergoing hypoxia and reoxygenation demonstrated $63 \%$ to $83 \%$ and $78 \%$ to $87 \%$ reductions, respectively, in MyD88 and TLR2 expression, respectively, compared with noncoding siRNA controls (Figure 2). TLR4 sequence-2, TLR2 sequence-1, and MyD88 sequence-3 were deemed most efficacious at achieving knockdown and used for subsequent experiments. Ten nanomolar TLR4 siRNA sequence- 2 treatment was the lowest dose with a strong knockdown efficacy $(80 \%)$ and was used for all subsequent in vivo studies (Figure 3). In vitro, we performed dose-response experiments with TLR4 concentrations of 


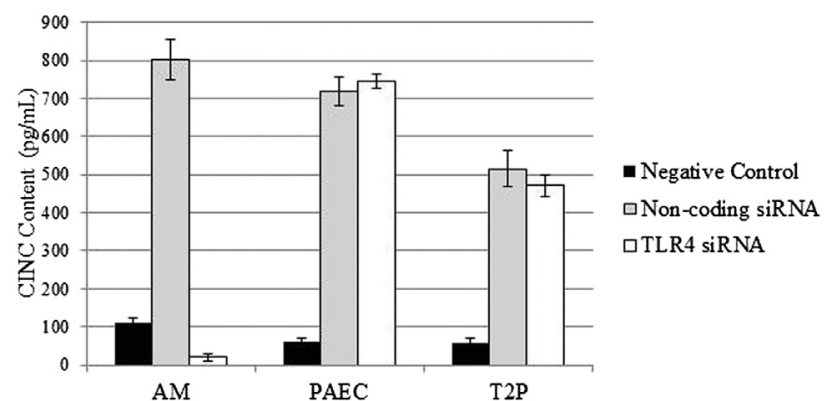

FIGURE 5. CINC production after TLR4 knockdown in cultured AM, PAEC, and T2P. In cultured AMs, PAECs, and T2Ps treated with noncoding siRNA controls before undergoing hypoxia and reperfusion, there was a 7-fold, 12-fold, and 9-fold increase in CINC production, respectively, compared with negative controls that did not undergo hypoxia and reoxygenation. CINC production was reduced by $98 \%$ in AMs treated with TLR4 siRNA before undergoing hypoxia and reperfusion compared with noncoding siRNA controls $(\mathrm{n}=12$ wells per group). However, CINC production was not significantly changed in PAECs ( $4 \%$ increase, $P>.05)$ or T2Ps ( $9 \%$ reduction, $P>.05$ ) treated with TLR4 siRNA before undergoing hypoxia and reperfusion compared with noncoding siRNA controls ( $\mathrm{n}=12$ wells per group). CINC, Cytokine-induced neutrophil chemoattractant; $A M$, alveolar macrophage; $P A E C$, pulmonary artery endothelial cell; $T 2 P$, type 2 pneumocyte; siRNA, short interfering RNA; $T L R$, toll-like receptor.

$10,50,100,500$, and $1000 \mathrm{pM}$ and observed similar knockdown efficacy at TLR4 siRNA concentrations of $100 \mathrm{pM}$ (data not shown).

All 3 markers of lung injury were significantly increased in noncoding siRNA controls after undergoing ischemia-reperfusion compared with negative controls (Figure 4). However, with TLR4 siRNA treatment before
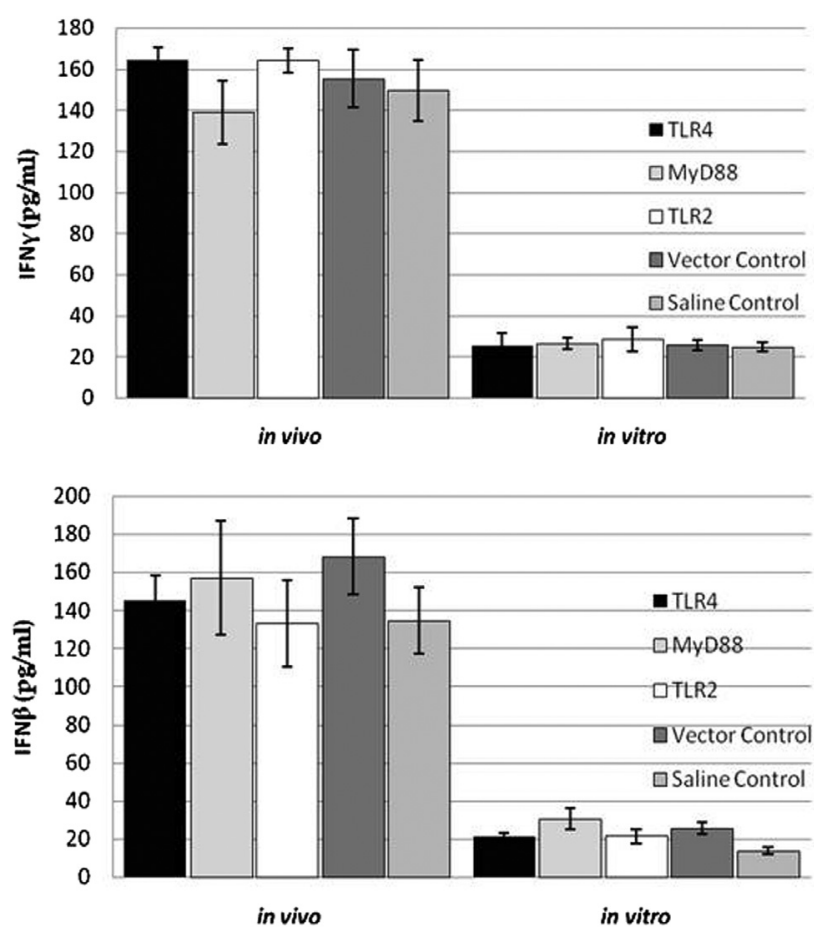

FIGURE 7. IFN production after siRNA administration. There was no significant difference $(P>.05)$ in IFN- $\gamma$ or IFN- $\beta$ production among rats treated with TLR4, MyD88, or TLR2 siRNA in cationic lipid vector, lipid vector alone, or normal saline alone at 1,6 , or 24 hours after treatment ( $\mathrm{n}=3,6$-hour data shown). In addition, there was no significant difference $(P>.05)$ in IFN- $\gamma$ or IFN- $\beta$ production by AMs treated with TLR4, MyD88, or TLR2 siRNA in cationic lipid vector, lipid vector alone, or normal saline alone at 1,6 , or 24 hours after treatment $(n=12$ wells per group, 6-hour data shown). IFN, Interferon; TLR, toll-like receptor; MyD88, myeloid differentiation factor 88 .

undergoing ischemia-reperfusion, all 3 markers of lung injury were significantly reduced, compared with noncoding siRNA controls (Figure 4). CINC production was significantly increased in primary cell cultures treated with noncoding siRNA controls before undergoing hypoxia and reoxygenation production compared with negative controls (Figure 5). However, CINC production was reduced only in AM, not PAEC or T2P, treated with TLR4 siRNA before undergoing hypoxia and reoxygenation compared with noncoding siRNA controls (Figure 5). TLR2 expression was present and unaffected in AM, PAEC, and T2P eluted from rats treated with TLR4 siRNA compared with noncoding siRNA controls as assessed by relative optical densitometry (Figure 6). There was no significant difference in IFN $\gamma$ or IFN $\beta$ production among rats or AM treated with TLR4, MyD88, or TLR2 siRNA in cationic lipid vector, lipid vector alone, or normal saline alone at 1,6 , or 24 hours after treatment (Figure 7, 6-hour data shown). 


\section{DISCUSSION}

This study establishes the cell-specific uptake of intravenously administered siRNA in the AM in the lung and our ability to transfect primary cell cultures of AM, PAEC, and T2P, and validates this novel approach for target gene knockdown in our model of LIRI. In addition, we have demonstrated reduced expression of the target gene using multiple unique siRNA sequences both in vivo and in vitro, as well as the specificity of the siRNA to reduce expression of only the target gene. Furthermore, we demonstrated that knockdown of TLR4 was associated with reduction in multiple markers of lung injury in vivo and decreased CINC production by AM. Dose-response experiments identified the lowest, most effective dose of siRNA to minimize OTEs. Finally, we demonstrated that siRNA administration was not associated with IFN production in vivo or in vitro. Taken together, these results significantly increase the confidence with which the observed phenotype can be ascribed to knockdown of the target protein and provide a powerful tool for studying the central role of the AM in the development of LIRI.

The validation of siRNA use in our model includes not only our results but also our experimental design and methodological considerations. Although the exact chemical modifications to Stealth siRNA (Invitrogen) are proprietary, the specific siRNA molecules chosen avoid any known stretches of RNA that can induce an IFN response, ${ }^{23}$ contain no sequence homology to each other when multiple siRNAs were used against the same target, and include chemical modifications ensuring that only the antisense strand of the siRNA duplex is processed by the endogenous RNA interference machinery. We not only demonstrated that the target protein was reduced in vivo and in vitro, a critical component of siRNA validation, but also did so with multiple nonoverlapping sequences and demonstrated that TLR2 was not affected by TLR4 siRNA, thereby confirming specificity and addressing a concern that siRNA can affect expression of nontarget TLR. ${ }^{26}$ Determining the lowest effective dose of siRNA was a significant component of these experiments, considering siRNA concentrations in excess of $100 \mathrm{nM}$ frequently produce nonspecific OTEs, whereas concentrations less than $20 \mathrm{nM}$, and especially less than $10 \mathrm{nM}$, rarely produce OTEs. ${ }^{26,27}$ Induction of the IFN response can result from siRNA itself or even its vector; therefore, regardless of any dose, design, or manufacturer modifications, measurement of in vivo and in vitro IFN production is essential and notably was not increased in our experiments. These steps, the appropriate design of siRNA, the experimental design considerations, and the confirmation of specificity, localization, and observed phenotype all contribute to the overall validation of siRNA use in our model of LIRI.

Intravenous siRNA administration has been repeatedly shown to transfect resident tissue inflammatory cells, particularly mononuclear cells, including the $\mathrm{AM},{ }^{7,10-12}$ whereas studies using intratracheal or intranasal siRNA administration have demonstrated inconsistent, erratic, or nonspecific cellular uptake of siRNA. ${ }^{28,29}$ Having previously identified the AM as the key coordinating cell type early in LIRI, ${ }^{9}$ the intravenous approach for siRNA administration provided a likely, and subsequently correct, route for targeted, cell-specific knockdown in the AM in vivo. Emerging methods for targeted intratracheal siRNA administration to the AM are under development, ${ }^{30}$ but if these potential approaches are successful, the validation strategy created for our study would be applicable, relevant, and translatable.

Investigating LIRI requires not only pertinent and appropriate animal models but also cell culture models to discretely map critical cell signaling events that would otherwise be cumbersome or impossible to do in vivo. Although in vitro studies fall short of reconstituting complex in vivo interactions, they serve as an important complement to the animal studies to characterize critical signaling events. Our warm hilar occlusion model is specific and reliable for the study of mechanisms of injury related to warm atelectatic ischemia-reperfusion. Our model is not only relevant but also addresses an important clinical problem that has a predictable time of onset. This is in strong contrast to most types of acute inflammatory lung injury and allows for practical pretreatment and intervention. Difficulty with target cell transfection has been one of the primary deterrents to the broader in vivo application of siRNA to other model systems, including myocardial and cerebral systems. ${ }^{6-8}$ The overall validation strategy used in this study nonetheless confirms siRNA technology to be particularly well suited for application in LIRI.

\section{CONCLUSIONS}

The immune-compromised lung transplant recipient faces numerous challenges after implantation, LIRI being one of the earliest and most significant, with the potential to affect both acute and chronic outcomes. LIRI has a predictable onset and increasingly an identifiable set of molecular signaling events that have recognized activation sequences. The transient nature of siRNA-mediated target mRNA degradation is ideally positioned for application in the setting of LIRI, with the ability to knock down the target gene of interest during the most injurious phase of LIRI, only to have that protein expression reconstitute to participate in normal innate immune resistance to 
infection and rejection. The simplicity of siRNA belies the complicated validation tactics developed and used in our model, necessary when using this technology, but the advantages and potential translatability of siRNA in the setting of LIRI justify its ongoing examination and advancement.

\section{Conflict of Interest Statement}

Authors have nothing to disclose with regard to commercial support.

\section{References}

1. Elbashir SM, Harborth J, Lendeckel W, Yalcin A, Weber K, Tuschl T. Duplexes of 21-nucleotide RNAs mediate RNA interference in cultured mammalian cells. Nature. 2001;411:494-8.

2. Dorsett Y, Tuschl T. siRNAs: applications in functional genomics and potential as therapeutics. Nat Rev Drug Discov. 2004;3:318-29.

3. Fire A, Xu S, Montgomery MK, Kostas SA, Driver SE, Mello C. Potent and specific genetic interference by double-stranded RNA in Caenorhabditis elegans. Nature. 1998;391:806-11.

4. Meister G, Tuschl T. Mechanisms of gene silencing by double-stranded RNA. Nature. 2004:431:343-9.

5. Dykxhoorn DM, Novina CD, Sharp PA. Killing the messenger: short RNAs that silence gene expression. Nat Rev Mol Cell Biol. 2003;4:457-67.

6. Bradley SP, Kowalik TF, Rastellini C, da Costa MA, Bloomenthal AB, Cicalese L, et al. Successful incorporation of short-interfering RNA into islet cells by in situ perfusion. Transplant Proc. 2005;37:233-6.

7. Sioud M, Sorensen DR. Cationic liposome-mediated delivery of siRNAs in adult mice. Biochem Biophys Res Commun. 2003;312:1220-5.

8. Larson SD, Jackson LN, Chen LA, Rychahou PG, Evers BM. Effectiveness of siRNA uptake in target tissues by various delivery methods. Surgery. 2007; 142:262-9.

9. Naidu BV, Woolley SM, Farivar AS, Thomas R, Fraga CH, Goss CH, et al. Early tumor necrosis factor-alpha release from the pulmonary macrophage in lung ischemia-reperfusion injury. J Thorac Cardiovasc Surg. 2004;127:1502-8.

10. Sorensen DR, Leirdal M, Sioud M. Gene silencing by systemic delivery of synthetic siRNAs in adult mice. J Mol Biol. 2003;327:761-6.

11. Jing Y, Shishkov A, Ponnappa BC. Inhibition of tumor necrosis factor alpha secretion in rat Kupffer cells by siRNA: in vivo efficacy of siRNA-liposomes. Biochim Biophys Acta. 2008;1780:34-40.

12. Sakurai F, Terada T, Mayuyama M, Watanabe M, Yamashita F, Takakura Y, et al. Therapeutic effect of intravenous delivery of lipoplexes containing the interferon-beta gene an poli I: poly $\mathrm{C}$ in a murine lung metastasis model. Cancer Gene Ther. 2003;10:661-8.

13. Angel LF, Levine DJ, Restrepo MI, Johnson S, Sako E, Carpenter A, et al. Impact of a lung transplantation donor-management protocol on lung donation and recipient outcomes. Am J Respir Crit Care Med. 2006;174:710-6.
14. King RC, Binns OA, Rodriguez F, Kanithanon RC, Daniel TM, Spotnitz WD, et al. Reperfusion injury significantly impacts clinical outcome after pulmonary transplantation. Ann Thorac Surg. 2000;69:1681-5.

15. Fiser SM, Tribble CG, Long SM, Kaza AK, Kern JA, Jones DR, et al. Ischemia-reperfusion injury after lung transplantation increases risk of late bronchiolitis obliterans syndrome. Ann Thorac Surg. 2002;73:1041-8.

16. de Perrot M, Liu M, Waddell TK, Keshavjee S. Ischemia-reperfusion-induced lung injury. Am J Respir Crit Care Med. 2003;167:490-511.

17. Wolf PS, Merry HE, Farivar AS, McCourtie AS, Mulligan MS. Stress-activated protein kinase inhibition to ameliorate lung ischemia reperfusion injury. J Thorac Cardiovasc Surg. 2008;135:656-65.

18. Naidu BV, Krishnadasan B, Farivar AS, Woolley SM, Thomas R, Van Rooijen N, et al. Early activation of the alveolar macrophage is critical to the development of lung ischemia-reperfusion injury. J Thorac Cardiovasc Surg. 2003;126:200-7.

19. Akira S, Kawai T. TLR signaling. Semin Immunol. 2007;19:24-32.

20. Jeyaseelan S, Manzer R, Young SK, Yamamoto M, Akira S, Mason RJ, et al. Toll-IL-1 receptor domain-containing adaptor protein is critical for early lung immune responses against Escherichia coli lipopolysaccharide and viable Escherichia coli. J Immunol. 2005;175:7484-95.

21. Jackson AL, Burchard J, Schelter J, Chau BN, Cleary M, Lim L, et al. Widespread siRNA "off-target" transcript silencing mediated by seed region sequence complementarity. RNA. 2006;12:1179-87.

22. Fedorov Y, Anderson EM, Birmingham A, Reynolds A, Karpilow J, Robinson K, et al. Off-target effects by siRNA can induce toxic phenotype. RNA. 2006;12: 1188-96.

23. Judge AD, Sood V, Shaw JR, Fang D, McClintock K, MacLachlan I. Sequence-dependent stimulation of the mammalian innate immune response by synthetic siRNA. Nat Biotechnol. 2005;23:457-62.

24. Naidu BV, Krishnadasan B, Byrne K, Farr AL, Rosengart M, Verrier ED, et al. Regulation of chemokine expression by cyclosporine A in alveolar macrophages exposed to hypoxia and reoxygenation. Ann Thorac Surg. 2002; 74:899-905.

25. Dobbs LG. Isolation and culture of alveolar type II cells. Am J Physiol. 1990; 258(4 Pt 1):L134-47.

26. Persengiev SP, Zhu X, Green MR. Nonspecific concentration dependent stimulation and repression of mammalian gene expression by small interfering RNAs (siRNAs). RNA. 2004;10:12-8.

27. Semizarov D, Frost L, Sarthy A, Kroeger P, Halbert D, Fesik S. Specificity of short interfering RNA determined through gene expression signatures. Proc Natl Acad Sci U S A. 2006;100:6347-52.

28. Miyawaki-Shimizu K, Predescu D, Shimizu J, Broman M, Predescu S, Malik AB. siRNA-induced caveolin-1 knock-down in mice increases lung vascular permeability via the junctional pathway. Am J Physiol Lung Cell Mol Physiol. 2005;290:L405-13.

29. Lomas-Neira JL, Chung CS, Wesche DE, Perl M, Ayala A. In vivo gene silencing (with siRNA) of pulmonary expression of MIP-2 versus KC results in divergent effects on hemorrhage-induced neutrophil-mediated septic acute lung injury. J Leukoc Biol. 2005;77:846-53.

30. Durcan N, Murphy C, Cryan SA. Inhalable siRNA: potential as a therapeutic agent in the lungs. Mol Pharm. 2008;5:559-66.

Key Words: lung reperfusion injury, siRNA, TLR4, TLR2 